\title{
Support for a Three-Item Questionnaire Prior to Spinal Surgery: A Health-Related Quality of Life Outcome Study
}

\author{
Kelly Anne Thomas ${ }^{1}$ Cara Sedney ${ }^{2}$ Richard Gross ${ }^{3}$ \\ ${ }^{1}$ Department of Counseling, Rehabilitation Counseling, and \\ Counseling Psychology, West Virginia University, Morgantown, \\ West Virginia, United States \\ ${ }^{2}$ Department of Neurosurgery, West Virginia University, \\ Morgantown, West Virginia, United States \\ ${ }^{3}$ Department of Behavioral Medicine and Psychiatry, West Virginia \\ University, Morgantown, West Virginia, United States
}

\begin{abstract}
Address for correspondence Cara Sedney, MD, Neurosurgery Clinic, West Virginia University, 1 Medical Center Drive, Morgantown, WV 26505, United States (e-mail: csedney@hsc.wvu.edu).
\end{abstract}

Abstract

Keywords
- health-related quality
of life
- coping
- questionnaire
- pain
- surgery

Objective Elective lumbar and cervical operations are becoming more common in the United States. Additionally, there is a movement in the literature and clinical practice to discover short versions of longer measures as a way to anticipate an outcome. This study aims to provide neurosurgeons in practice with a three-item questionnaire that can guide referrals to psychological services presurgery. Ultimately, results could lead to an improvement in health-related quality of life (HRQoL) postspinal surgery.

Methods This quantitative-descriptive, survey-based design with a retrospective chart review component followed 47 patients at baseline ( $N=47), 3$ months $(N=20)$, 6 months $(N=31)$, and 1 year $(N=19)$. A single item from the Coping Strategies Questionnaire, the Survey of Pain Attitudes, and the Tampa Scale of Kinesiophobia were utilized in the three-item questionnaire as a baseline measure. Patient-Reported Outcomes Measurement Information System Global Health measured HRQoL outcome at all time points. A linear regression model was conducted to predict mental health QoL postspinal surgery.

Results This measure can predict mental health QoL outcomes up to 3-month postsurgery. Six-month and 1-year follow-ups are statistically inconclusive.

Conclusion Individuals who are undergoing spinal surgery show lower mental health QoL outcome at baseline and 3-month postsurgery when responses on a three-item questionnaire are elevated. Limitations and future directions are discussed.

\section{Introduction}

The number of individuals electing to have spinal surgery is rising. ${ }^{1,2}$ Specifically, in the United States, spinal surgery continues to increase at faster rates when compared with other musculoskeletal procedures.,4 Despite these upward trends, a key factor to consider are the high rates of poor pain-related outcome postsurgery. ${ }^{5,6}$ One example of a poor outcome postspinal surgery is a continued experience of neuropathic pain, which may result in a diagnosis of failed

DOI https://doi.org/ 10.1055/s-0039-3400348 ISSN 0976-3147. back syndrome. ${ }^{7}$ Consequently, there is a current trend in the literature to determine factors that relate to a positive or negative outcome for these individuals. One construct that literature has considered is how psychological variables affect surgical recovery. ${ }^{8,9}$

Lethem et $\mathrm{al}^{10}$ suggested that pain-related fears can increase disability and decrease the ability to recover postinjury. Examples of these pain-related fears that affect pain severity postsurgery are pain catastrophizing, kinesiophobia, and harm. ${ }^{11-15}$ Pain catastrophizing is defined as a negative 
orientation toward current or future pain experiences which may result in maladaptive coping strategies. ${ }^{11}$ Additionally, kinesiophobia refers to a fear of movement, which has been noted in the literature as a significant factor in rehabilitation postspinal surgery. ${ }^{12-14} \mathrm{~A}$ third factor that contributes to chronic pain disability is harm, which is the belief that one should avoid future experiences that have the potential to cause physical harm. ${ }^{15}$

While these psychological constructs have been shown to be important, a barrier to use has historically been the time demands for administration and scoring of lengthy inventories. Recently, there has been an effort to develop short-form versions of well-validated measures to improve clinical utility. For example, Jensen et al have created one- and two-item measures from scales such as the Survey of Pain Attitudes (SOPA) $)^{15}$ and the Coping Strategies Questionnaire (CSQ), ${ }^{16}$ which provide clinicians and researchers the ability to utilize short forms of longer constructs. ${ }^{17}$

In assessing outcome for postoperative lumbar and cervical surgery, health-related quality of life (HRQoL) has been widely used as a way to measure the effects that surgery or diagnosis has on physical, mental, and social functioning. ${ }^{18,19}$ In a pilot study of 57 individuals receiving lumbar surgery, patients, 3-month postsurgery, reported overall higher HRQoL, reporting higher functionality and lower pain intensity. ${ }^{20}$ However, research on HRQoL outcome has been challenging. Therefore, the National Institute of Health has developed the Patient-Reported Outcomes Measurement Information System (PROMIS) to address limitations from past "legacy" measures (i.e., Oswestry disability index, visual analog scale). ${ }^{21,22}$ In a recent study comparing the legacy measures versus PROMIS function scores, there were strong correlates that further validated PROMIS as a HRQoL outcome measure for lumbar microdiscectomy. ${ }^{23}$

The current study investigates the utility of a three-item measure for preoperative use which assesses pain-related catastrophizing, fear of movement, and fear of harm as a way to predict HRQoL postspinal surgery. It is hypothesized that the three-item measure of pain-related distress will be predictive of poorer mental health QoL postsurgery. The overall aim of the study is to provide neurosurgeons and other clinicians with the opportunity to provide their patients with services needed prior to spinal surgery to improve HRQoL postsurgery.

\section{Methods}

\section{Design}

This study utilizes a quantitative-descriptive, survey-based design with a retrospective chart review component. ${ }^{24}$ Data sources include a retrospective chart review to gather information on patient demographics, type of surgery (e.g., cervical vs. lumbar) and a three-item questionnaire. Additionally, PROMIS questionnaires were distributed to measure HRQoL outcome. A linear multiple regression was utilized to determine the predictability of the three-item questionnaire and psychological distress (HRQoL) pre- and postspinal surgeries.

\section{Sample}

The use of a $G^{*}$ Power 3.1 software determined the number of participants needed for statistical power. Once utilizing the recommendations made by Faul et $\mathrm{al}^{25}$ on appropriate calculations for determining a significant effect size for a linear multiple regression: fixed model, $R^{2}$ increase, the total sample size was determined to be a minimum $37(N=37)$ for statistical significance. This was based on a large effect size of Cohen's $f^{2}=0.35$, a power ( $1-\beta$ error probability) of 0.95 , and an $\alpha(\alpha)$ of $0.05 .{ }^{25}$ Participants older than the age of 18 years and had lumbar or cervical surgery between the years 2016 and 2018 at a mid-Atlantic medical facility were included. This mid-Atlantic facility is a rural-referral center, and serves a high volume of individuals living in rural communities. One hundred and seventeen participants were originally included in this study. There were 66 individuals who did not complete or partially completed questionnaires during intake which excluded them from this study. Therefore, 47 participants included in this study at baseline were between the ages of 31 and 79 years ( mean $=56$, standard deviation $[S D]=11.80$ ). Twenty-five of these individuals identified as male, and 22 identified as female. Twenty participants were cervical surgery patients, and 27 were lumbar surgery patients at baseline. Data collection occurred at baseline, 3-month, 6-month, and 1-year follow-ups.

\section{Instruments}

A three-item questionnaire was produced utilizing one catastrophizing item from the CSQ one harm item from the SOPA, and one item measuring the fear of movement from the Tampa Scale of Kinesiophobia (TSK) ( - Fig. 1). ${ }^{15,16,26}$ Jensen et al validated one- and two-item measures of pain beliefs. ${ }^{17}$

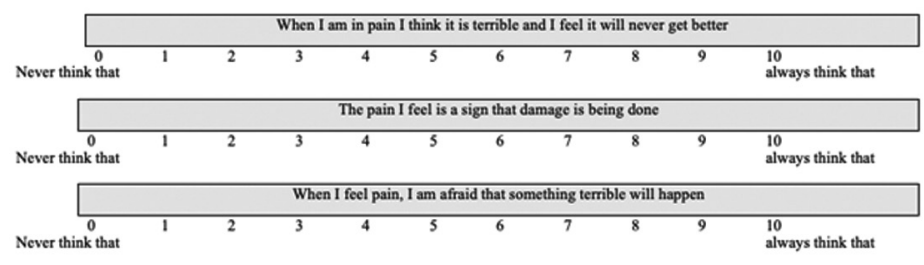

Fig. 1 Three-item questionnaire completed at baseline. Includes single item from the Coping Strategies Questionnaire, the Survey of Pain Attitudes, and the Tampa Scale of Kinesiophobia. 
Specifically, the single catastrophizing item on the CSQ and the harm item on the SOPA are noted to be a reliable single-item measures. ${ }^{17}$ An additional single item from the TSK was selected based on clinical face validity to as a measure of fear of movement. The TSK is noted to have good test-retest reliability, concurrent validity, and predictive validity. ${ }^{26,27}$ PROMIS was utilized as an outcome measure to quantify variables such as pain, fatigue, physical functioning, emotional distress, and social functioning. Ten variables were applied for data analysis from PROMIS data. These categories include (1) QoL vitality, (2) QoL social functioning, (3) QoL role physical, (4) QoL role emotional, (5) QoL physical functioning, (6) QoL physical component summary, (7) QoL mental health, (8) QoL mental component summary, (9) QoL general health, and (10) QoL bodily pain.

\section{Data Collection}

Participants were patients at a mid-Atlantic medical facility who were referred by a physician for a lumbar or cervical operation. Patients consented prior to the administration of outcome measures. A retrospective chart review was completed to gather information on previous medical history, referral source, age at surgery, and sex. At baseline, patients completed a three-item questionnaire along with an intake packet. All PROMIS measures were completed on a tablet during their regularly scheduled clinic visits and were stored in an electronic medical record. Data collection was acquired at baseline, 3 months, 6 months, and 1 year (-Fig. 2). All procedures of this study were in accordance with the WVU Institutional Review Board.

\section{Data Analysis}

This quantitative-descriptive, survey-based design first utilized a retrospective chart-review component to collect information on demographics. ${ }^{24}$ Means and SDs were calculated for each demographic. For analysis of quantitative and descriptive data, SPSS version 24 (IBM Corp., Armonk, New York, United States) was used. Individual scores of the three-item questionnaire were calculated along with a total score at baseline. Individual scores of the 10 QoL PROMIS Global measures were calculated at each time point (baseline, 3-month, 6-month, and 1-year posttreatment). The hypothesis being tested includes, $\mathrm{H}_{1}$ : Elevated scores on a three-item questionnaire completed at baseline will predict lower scores in areas related to mental health QoL postsurgery. This hypothesis was analyzed with a linear multiple regression: fixed model, $R^{2}$ increase.

\section{Results}

Of the original 117 participants, 47 completed baseline measures, 20 completed outcome measures at 3 months, 31 completed outcome measures at 6 months, and 19 completed outcome measures at 1 year. Data were split between the time points to compare QoL postsurgery. To control for the predictor variables (single items and total score on the three-item questionnaire), the mean score of each item was subtracted from the individual scores.

\section{Single-Item Catastrophizing Question from the CSQ}

After controlling for question 1 (Q1), an analysis of the regression model shows that a single-item catastrophizing question
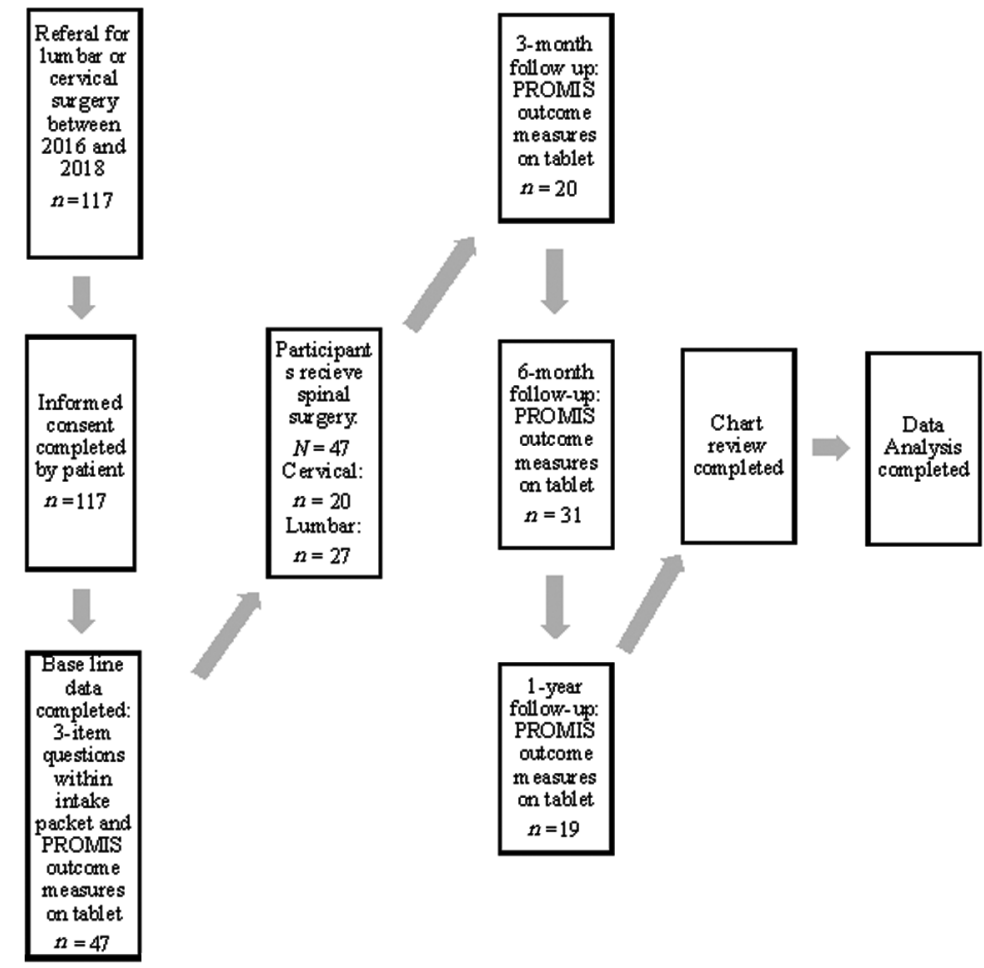

Fig. 2 Flow diagram depicting demographics and each stage of data collection. PROMIS, Patient-Reported Outcomes Measurement Information System. 
can significantly predict HRQoL at baseline $F(8,38)=2.677$, $p=0.019, R^{2}=0.360$ and 3 months $F(8,11)=4.094, p=0.017$, $R^{2}=0.749$, but not at 6 months $F(8,22)=0.399, p=0.909$, $R^{2}=0.127$ and 1 year $F(8,9)=1.487, p=0.283, R^{2}=0.569$ postspinal surgery. There were several factors that contributed to this prediction. First, at baseline, Q1 predicted QoL vitality $(\beta=0.4346, p=0.034)$ and mental health $(\beta=0.896$, $p=0.001)$. At 3-month postspinal surgery, the single-item catastrophizing question predicted QoL role emotional ( $\beta=0.543, p=0.014$, and QoL bodily pain $(\beta=0.650, p=0.043)$.

\section{Single-Item Harm Question from the SOPA}

After controlling for question 2 (Q2) on the three-item questionnaire, an analysis of the regression model shows this single-item harm question can significantly predict HRQoL at baseline $F(8,38)=2.441, p=0.031, R^{2}=0.339$, but not at 3 months $F(8,11)=2.106, p=0.126, R^{2}=0.605 ; 6$ months $F(8,22)=1.295, p=0.296, R^{2}=0.320$; or 1 year $F(8,9)=$ 3.012, $p=0.060, R^{2}=0.728$. At baseline, $\mathrm{Q} 2$ predicted $\mathrm{QoL}$ mental health $(\beta=0.544, p=0.046)$.

\section{Single-Item Fear-Avoidance Question from the TSK}

An analysis of the regression model was conducted after controlling for the third question (Q3) on the three-item questionnaire. The model summary suggests a fear-avoidance question on the TSK can significantly predict HRQoL at baseline $F(8,38)=5.098, p=0.000, R^{2}=0.518$ and 3 months $F(8,11)=6.328, p=0.003, R^{2}=0.822$, but not at 6 months $F(8,22)=1.625, p=0.175, R^{2}=0.371$ or 1 year $F(8,9)=$ $2.608, p=0.088, R^{2}=0.699$. There were several factors that contributed to this prediction. First, at baseline, Q3 predicted QoL vitality $(\beta=0.460, p=0.011)$ and mental health ( $\beta=0.812, p=0.001)$. At 3-month postspinal surgery, the single-item fear-avoidance question predicted QoL role emotional $(\beta=0.743, p=0.001)$.

\section{Total Score of the Three-Item Questionnaire}

Finally, a total score of the three-item questionnaire was computed through the summation of answers on questions 1, 2, and 3 for each individual included in this study $(N=47)$. An analysis of the regression model was conducted after controlling for the total score on the three-item questionnaire. The model summary suggests the total score can significantly predict HRQoL at baseline $F(8,38)=5.618, p=0.000, R^{2}=$ 0.542 and 3 months $F(8,11)=3.626, p=0.026, R^{2}=0.725$, but not at 6 months $F(8,22)=1.021, p=0.450, R^{2}=0.271$ and 1 year $F(8,9)=2.984, p=0.062, R^{2}=0.726$. There were HRQoL variables that contributed to this prediction. First, at baseline, the total score predicted QoL vitality $(\beta=0.528, p=0.003)$ and mental health $(\beta=0.928, p=0.000)$. At 3 -month postspinal surgery, the total score of the three-item questionnaire predicted QoL role emotional $(\beta=0.663, p=0.006)$.

\section{Discussion}

The aim of this study was to provide neurosurgeons and other clinicians with a three-item questionnaire that would help navigate patients to proper psychological services prior to cervical or lumbar operation to improve HRQoL postsurgery. The findings show that this three-item questionnaire can be applied at baseline as a way to predict mental health outcomes up to 3-month postspinal surgery. Q1 (single-catastrophizing item from CSQ) ${ }^{16,17}$ was predictive of vitality and mental health at baseline, and role emotional and bodily pain at 3 months. Q2 (single-harm item from SOPA $)^{15,17}$ was predictive of mental health only at baseline but had no other significance 3 months and beyond. Q3 (single fear-avoidance item from TSK) ${ }^{26}$ was predictive of vitality and mental health at baseline, and role emotional at 3 months. Finally, the total score of all three items was predictive of mental health and vitality at baseline, and role emotional at 3-month postsurgery.

Of the 10 HRQoL measures included in the PROMIS data ([1]) QoL vitality, [2] QoL social functioning, [3] QoL role physical, [4] QoL role emotional, [5] QoL physical functioning, [6] QoL physical component summary, [7] QoL mental health, [8] QoL mental component summary, [9] QoL general health, and [10] QoL bodily pain), vitality, role emotional, mental health, and bodily pain were the four outcomes that were correlated to these four predictors (Q1, Q2, Q3, and total score) both separately and together. Therefore, the other HRQoL variables did not relate with high scores on the threeitem questionnaire. These findings suggest that this threeitem questionnaire should only be used to predict mental health for patients at baseline and 3-month postspinal surgery. These findings support the initial hypothesis.

Individuals who score high on this measure (scores range $=0-30,0=$ low score and $30=$ high score) should be considered as a candidate for mental health counseling prior to spinal surgery. This preadmission counseling prior to surgery could inform patients of the surgical intervention, protocols of the hospital, and has been noted to facilitate recovery and pain relief postsurgery. ${ }^{28,29}$ Furthermore, a psychological evaluation prior to surgery could provide surgeons with valuable information such as assessing mental health functioning, determining comorbid factors that could jeopardize the client's recovery, determine risk factors such as adherence to a postoperative treatment plan, and provide the patient with valuable coping skills prior to surgery. ${ }^{30}$ Additionally, utilizing this instrument promotes interdisciplinary care. Literature has supported the notion of participating in interdisciplinary care when caring for a patient due to its movement toward a biopsychosocial approach. ${ }^{31-33}$

This study has several limitations that are necessary to address. The most prominent limitation is the low number of responses at 3-month, 6-month, and 1-year postsurgery. After running a $G^{*}$ Power analysis with a large effect size of Cohen's $f^{2}=0.35$, a power ( $1-\beta$ error probability) of 0.95 , and an $\alpha$ of 0.05 , the minimum number needed was 37 participants. This number was reached at baseline $(N=47)$ and not at the 3-month, 6-month, and 1-year follow-ups. This limitation threatens the external validity of the study by making it difficult to consider this sample as representative for postsurgical patients. ${ }^{34}$ However, significant results were still discovered in these data at baseline and 3-month postspinal surgery. A 
second limitation threatens the internal validity of this study through the use of a single fear-avoidance item taken from the TSK. While the TSK has supported as a reliable and valid measure, ${ }^{26,27}$ the single fear-avoidance item has not been studied independently. However, the single item from the CSQ and SOPA have been noted to be reliable single-item measures. ${ }^{17}$ Lambert and Hill described an additional limitation to outcome-based research is the difficulty in determining if statistical findings are a good indicator of clinical outcomes. ${ }^{35}$

These limitations provide readers with several recommendations for future directions. First, validation of the individual items on the TSK could provide researchers and clinicians with the opportunity to utilize single items in future publications and clinical practice. Second, increasing the number of pre- and postsurgical patients could provide lesser threats to external validity. Third, a follow-up to this study on the effectiveness of an evaluation with a pain psychologists or other mental health professional for individuals who score high on the three-item questionnaire would provide valuable feedback to this study and validate the measure as well as the clinical significance preadmission pain counseling can have on mental health QoL postspinal surgery.

\section{Conclusion}

Although current trends in clinical research are to produce short-form versions of existing measures, to the best of the authors' knowledge, this study is the first to utilize a threeitem questionnaire for use as a predictive measure in HRQoL postspinal surgery. Results indicate that individuals who are undergoing spinal surgery show lower mental health QoL outcome at baseline and 3-month postsurgery when responses on a three-item questionnaire are elevated.

\section{Funding \\ None.}

\section{Conflict of Interest}

None declared.

\section{Acknowledgments}

The authors thank Sherri Davis for her involvement in data collection, and Dr. John France for his encouragement and support of the project.

\section{References}

1 Baird EO, Egorova NN, McAnany SJ, Qureshi SA, Hecht AC, Cho SK. National trends in outpatient surgical treatment of degenerative cervical spine disease. Global Spine J 2014;4(3):143-150

2 Martin BI, Mirza SK, Spina N, Spiker WR, Lawrence B, Brodke DS. Trends in lumbar fusion procedure rates and associated hospital costs for degenerative spinal diseases in the United States, 2004-2015. Spine 2019;44(5):369-376

3 Deyo RA, Gray DT, Kreuter W, Mirza S, Martin BI. United States trends in lumbar fusion surgery for degenerative conditions. Spine 2005;30(12):1441-1445

4 Rajaee SS, Bae HW, Kanim LE, Delamarter RB. Spinal fusion in the United States: analysis of trends from 1998 to 2008. Spine 2012;37(1):67-76
5 Dvorak J, Gauchat MH, Valach L. The outcome of surgery for lumbar disc herniation. I. A 4-17 years' follow-up with emphasis on somatic aspects. Spine 1988;13(12):1418-1422

6 Kehlet H, Jensen TS, Woolf CJ. Persistent postsurgical pain: risk factors and prevention. Lancet 2006;367(9522):1618-1625

7 Cho JH, Lee JH, Song K-S, Hong J-Y. Neuropathic pain after spinal surgery. Asian Spine J 2017;11(4):642-652

8 Wilhelm M, Reiman M, Goode A, et al. Psychological predictors of outcomes with lumbar spinal fusion: a systematic literature review. Physiother Res Int 2017;22(2):e1648

9 Celestin J, Edwards RR, Jamison RN. Pretreatment psychosocial variables as predictors of outcomes following lumbar surgery and spinal cord stimulation: a systematic review and literature synthesis. Pain Med 2009;10(4):639-653

10 Lethem J, Slade PD, Troup JD, Bentley G. Outline of a fear-avoidance model of exaggerated pain perception-I. Behav Res Ther 1983;21(4):401-408

11 Gatchel RJ, Rollings KH. Evidence-informed management of chronic low back pain with cognitive behavioral therapy. Spine J 2008;8(1):40-44

12 Kori SH, Miller RP, Todd DD. Kinisophobia: a new view of chronic pain behavior. Pain Management 1990;3:35-43

13 Picavet HS, Vlaeyen JW, Schouten JS. Pain catastrophizing and kinesiophobia: predictors of chronic low back pain. Am J Epidemiol 2002;156(11):1028-1034

14 Svensson GL, Lundberg M, Östgaard HC, Wendt GK. High degree of kinesiophobia after lumbar disc herniation surgery: a cross-sectional study of 84 patients. Acta Orthop 2011;82(6):732-736

15 Jensen MP, Turner JA, Romano JM, Lawler BK. Relationship of pain-specific beliefs to chronic pain adjustment. Pain 1994;57(3):301-309

16 Rosenstiel AK, Keefe FJ. The use of coping strategies in chronic low back pain patients: relationship to patient characteristics and current adjustment. Pain 1983;17(1):33-44

17 Jensen MP, Keefe FJ, Lefebvre JC, Romano JM, Turner JA. Oneand two-item measures of pain beliefs and coping strategies. Pain 2003;104(3):453-469

18 Fairclough DL. Design and Analysis of Quality of Life Studies in Clinical Trials. Boca Raton: Chapman \& Hall/CRC; 2010

19 Abbott AD, Tyni-Lenné R, Hedlund R. The influence of psychological factors on pre-operative levels of pain intensity, disability and health-related quality of life in lumbar spinal fusion surgery patients. Physiotherapy 2010;96(3):213-221

20 Saban KL, Penckofer SM, Androwich I, Bryant FB. Healthrelated quality of life of patients following selected types of lumbar spinal surgery: a pilot study. Health Qual Life Outcomes 2007;5(1):71

21 Fidai MS, Saltzman BM, Meta F, et al. Patient reported outcome measurements information system and legacy patient-reported outcome measures in the field of orthopaedis: a systemic review. Arthroscopy 2018;34(2):605-614

22 Cella D, Yount S, Rothrock N, et al. PROMIS Cooperative Group. The Patient-Reported Outcomes Measurement Information System (PROMIS): progress of an NIH Roadmap cooperative group during its first two years. Med Care 2007;45(5, suppl 1):S3-S11

23 Khechen B, Haws BE, Patel DV, et al. PROMIS physical function score strongly correlates with legacy outcome measures in minimally invasive lumbar microdiscectomy. Spine 2019;44(6):442-446

24 Heppner PP, Wampold BE, Owen J, Thompson MN, Wang KT. Research Design in Counseling, 4th ed. Boston, MA: Cengage Learning; 2016

25 Faul F, Erdfelder E, Buchner A, Lang A-G. Statistical power analyses using $G^{*}$ Power 3.1: tests for correlation and regression analyses. Behav Res Methods 2009;41(4):1149-1160 
26 Miller RP, Kori SH, Todd DD. The Tampa scale. Clin J Pain 1991;7(1):51

27 Mintken PE, Cleland JA, Whitman JM, George SZ. Psychometric properties of the Fear-Avoidance Beliefs Questionnaire and Tampa Scale of Kinesiophobia in patients with shoulder pain. Arch Phys Med Rehabil 2010;91(7):1128-1136

28 Raju B, Reddy K. Are counseling services necessary for the surgical patients and their family members during hospitalization? J Neurosci Rural Pract 2017;8(1):114-117

29 Fearon KC, Ljungqvist O, Von Meyenfeldt M, et al. Enhanced recovery after surgery: a consensus review of clinical care for patients undergoing colonic resection. Clin Nutr 2005;24(3):466-477

30 Williams DA. The importance of psychological assessment in chronic pain. Curr Opin Urol 2013;23(6):554-559

31 Havelka M, Lucanin JD, Lucanin D. Biopsychosocial modelthe integrated approach to health and disease. Coll Antropol 2009;33(1):303-310
32 Guzmán J, Esmail R, Karjalainen K, Malmivaara A, Irvin E, Bombardier C. Multidisciplinary bio-psycho-social rehabilitation for chronic low back pain. Cochrane Database Syst Rev 2002; ( 1):CD000963

33 Stanos S, Houle TT. Multidisciplinary and interdisciplinary management of chronic pain. Phys Med Rehabil Clin N Am 2006;17(2):435-450,

34 Price JH, Murnan J. Research limitations and the necessity of reporting them. Am J Health Educ 2004;35(2):66-67

35 Lambert MJ, Hill CE. Assessing psychotherapy outcomes and processes. In: Bergin AE, Garfield SL, eds. Handbook of Psychotherapy and Behavior Change. Oxford, England: John Wiley \& Sons; 1994 72-113 